\title{
ARTICULACIÓN DE LA POLÍTICA EDUCATIVA Y FEDERALISMO EN MÉXICO
}

Oscar Mauricio Covarrubias MORENO ${ }^{1}$

Oscar Mauricio Covarrubias ROMERO ${ }^{2}$

RESUMEN: El propósito del presente artículo es analizar la política educativa de México a partir de la firma del Acuerdo para la Modernización de la Educación Básica en 1992. Se parte de la hipótesis de que el modelo de descentralización o Federalismo educativo que se instaura a través del Acuerdo Nacional, ha afectado la gobernabilidad del sistema educativo en su conjunto, y de manera particular, la capacidad de conducción de la Secretaría de Educación Pública como autoridad nacional.

PALABRAS CLAVE: Federalismo. Política educativa. Descentralización. Gobernabilidad. Sistema educativo. Política sectorial.

\section{Introducción}

El propósito del presente artículo es analizar la política educativa a partir de la firma del Acuerdo para la Modernización de la Educación Básica en 1992. El cual, constituye una referencia en la historia de la educación en México, porque representa el primer intento de reforma de carácter integral en el sentido de que, además de la reformulación curricular, la revaloración de la función magisterial y el impulso a la participación social, comprende el replanteamiento general de la organización y gestión del sistema educativo.

Se parte de la hipótesis de que el modelo de descentralización o Federalismo educativo que se instaura a través del Acuerdo Nacional, ha afectado la gobernabilidad del sistema educativo en su conjunto, y de manera particular, la capacidad de conducción de la Secretaría de Educación Pública como autoridad nacional responsable de fijar los objetivos, estrategias y líneas de acción de la política educativa, de medir resultados y de compensar diferencias. En otras palabras, de dirigir el sistema educativo hacia el objetivo de brindar educación de calidad para todos.

Para delimitar el objeto de estudio, debemos señalar que entre los numerosos

\footnotetext{
1 Secretario General de la Universidad del Desarrollo Empresarial y Pedagógico (UNIVDEP). Postdoctorales en Ciencias Políticas y Sociales. UNM - The University of New México. New Mexico Estados Unidos. 87131 - mauricio.covarrubias@univdep.edu.mx

2 Maestrante en Gobiernos y Asuntos Públicos en la Facultad de Ciencias Políticas y Sociales. Universidad Nacional Autónoma de México. Ciudad de México - México. 04510 mau_covas@hotmail.com
} 
factores que inciden en la gobernabilidad del sistema educativo, nuestro análisis quiere mostrar la desarticulación que padece la política educativa nacional, producto de la falta de coordinación entre las acciones y los niveles de gobierno, que se manifiesta a partir de la descentralización. De ninguna manera se busca argumentar en contra del federalismo como sistema de organización política, sino llamar la atención sobre la experiencia concreta del denominado Federalismo educativo en México, y de cómo las ventajas que supone la descentralización pueden malograrse por deficiencias en su concepción e implantación.

De acuerdo con este propósito, el ensayo se ha dividido en cuatro apartados. En el primero, se plantea el gobierno del sistema educativo como un problema teórico, político y práctico. En el segundo, se explora el significado de la noción de política educativa nacional en el contexto del modelo de descentralización existente, conocido como Federalismo educativo. El tercer apartado, se documentan los problemas de articulación de la política educativa en el federalismo. El cuarto apartado, realiza un deslinde entre el Programa Sectorial de Educación y la Política Nacional de Educación. Finalmente, se presentan las conclusiones.

\section{El gobierno del sistema educativo}

Después de dos décadas de aplicación de reformas a los sistemas educativos en diversos países de Iberoamérica, nos encontramos en condiciones de plantear la cuestión del gobierno del sistema educativo, como un problema teórico, político y práctico. Sin embargo, dado que éste no opera en el vacío, es preciso que ubiquemos previamente el tema, en el contexto de los problemas de gobierno que aquejan a la sociedad.

Hoy día la tarea de gobierno involucra complejas configuraciones e interacciones al interior de la administración pública y de ésta con su entorno, a través de una diversidad de conexiones que marcan el devenir de importantes políticas y programas gubernamentales. Los problemas públicos se han transformado en cuanto a su extensión, intensidad y conectividad. Suponen una mayor afectación territorial y poblacional, una acumulación de efectos, así como un aumento significativo en la cantidad de los factores involucrados. Podemos decir que a la ampliación en la escala de los fenómenos, corresponde también un aumento en la escala en que deben tomarse las decisiones (COVARRUBIAS, 2006).

La complejidad en los Estados, indica Claus Offe, más que en otro tipo de organizaciones, atenta contra su integridad y coherencia orgánica. A partir de la noción de 
"descomposition of state power by increase of functions", sostiene que el reclamo a los Estados de decisiones racionales, se explica por el hecho que la multiplicación de responsabilidades se acompaña por un aumento en las instancias, autoridades, y agentes administrativos implicados. Para Offe, esto produce una pluralización interior y una fragmentación de perspectivas departamentales dentro de la administración, una intensificación en los conflictos, y en general, un incremento en la imprevisibilidad de los resultados a largo plazo, así como en los supuestos efectos "sinergéticos" de políticas individuales que resultan casi imposibles de coordinar (OFFE, 1996). En nuestra opinión, lo anterior tiende a acentuarse en sistemas de gobierno federal, donde la administración pública representa un complicado sistema multiorganizacional diferenciado funcionalmente por unidades departamentales y sectores, verticalmente por niveles, y horizontalmente por territorios, de forma que la implementación de una política no atañe a una instancia en particular, sino a un conglomerado de ellas.

Bajo estas consideraciones, aunque la complejidad se hace presente en los diferentes ámbitos de la tarea gubernamental, es en el área social donde es más visible y consecuentemente más necesaria una gestión pública articulada. Las políticas de salud, seguridad pública, ambiental o la educativa, objeto de nuestro interés, por mencionar solo algunas, se encuentran relacionadas con problemáticas que demandan aunar esfuerzos entre diferentes organizaciones no sólo al interior de un mismo gobierno, sino con otros gobiernos implicados geográfica y jurisdiccionalmente. Se trata de cuestiones que no conocen de límites, ni se detienen ante ninguna barrera, cuyas causas se encuentran interrelacionadas o mutuamente determinadas. En tal sentido, los mapas político políticoadministrativos ya no son muy útiles para comprenderlos y menos aún para contenerlos.

La política educativa, es una cuestión que ilustra bien las dificultades para alcanzar una acción pública conjunta, en un asunto que requiere de la intervención de distintos niveles de gestión. Dada la estructura actual de los sistemas de educación en la mayoría de los países Iberoaméricanos, el desarrollo de una política general, supone difíciles interacciones dentro, y entre la administración pública educativa a nivel central, subnacional, municipal y escolar, lo mismo que entre ésta y su entorno.

Definir la noción de gobierno del sistema educativo impone considerar una serie de conceptos que asociados o contenidos en la propia idea, ayudan a precisar lo esencial de la misma. Dentro de los límites que impone la extensión del ensayo, como parte de este apartado se precisarán algunos conceptos que nos permitan plantear la conducción de los sistemas educativos como un problema de investigación. En primer lugar, nos 
referiremos a la categoría de sistema educativo. La noción de sistema ha estado presente en diversas concepciones sociológicas del fenómeno educativo, como herramienta conceptual ha contribuido a comprender la complejidad propia de la función educativa y de sus relaciones internas y externas.

Según Crozier (1969), el sistema educativo es un reflejo del sistema social, y constituye al mismo tiempo, el medio esencial de perpetuarse. Al referirse a los elementos característicos del sistema burocrático como el apego a reglas, las relaciones verticales, la especialización y la obediencia como virtud cardinal Crozier señala que estos patrones se ordenan alrededor del problema del control social y no pueden subsistir sino transmitidos y fortalecidos por la educación. Desde la teoría social de Luhmann, la educación no sólo es un sistema constitutivo, sino constituyente del sistema social (LUHMANN; EBERHARD, 1993). Nos parece importante recuperar en este punto la opinión de Sander (1974), quien considera que la utilización de la perspectiva de sistémica no solo es posible, sino indispensable para entender y desarrollar la acción educativa. Por la naturaleza de las funciones que tiene asignadas, la educación es uno de los componentes fundamentales de todo sistema social en las sociedades modernas.

Para nuestros propósitos, los vocablos de gobierno, gobernabilidad y gobernanza, también resultan esenciales. Se trata sin lugar a dudas, de términos “anudados" que se sugieren recíprocamente. Por ejemplo, el Diccionario de la Real Academia Española (2010) asocia el vocablo de gobernabilidad a la "cualidad de gobernable", y lo equipara al concepto de gobernanza como "arte o manera de gobernar" que se propone como objetivo el logro de un desarrollo económico, social e institucional duradero, promoviendo un sano equilibrio entre el Estado, la sociedad civil y el mercado de la economía. El diccionario también define a la gobernanza como la “Acción y efecto de gobernar o gobernarse.

La gobernanza remite a una forma de gobernar más cooperativa, distinta del antiguo modelo jerárquico, en el que las autoridades ejercían un poder soberano sobre los grupos y ciudadanos que constituyen la sociedad civil. De acuerdo con Mayntz, ahí donde se desarrollan redes de políticas, el gobierno deja de ser el centro director de la sociedad: 
un consenso negociado que facilita la formación de una política, que en el momento de su aplicación encuentre más aceptación que resistencia. (MAYNTZ, 2001).

Específicamente la gobernabilidad de los sistemas educativos, ha sido definida en la "Declaración de Concepción" de la Organización de Estados Iberoamericanos para la Educación, la Ciencia y la Cultura (1996) como la capacidad para atender las demandas de educación de la población escolar y de la sociedad en su conjunto, así como la aptitud para resolver los conflictos internos que se producen en su seno. De esta manera, la Declaración da cuenta de la emergencia del tema de la gobernabilidad de los sistemas educativos de Iberoamérica que, ya para entonces, contaban con diversas reformas en su haber.

Es importante mencionar que la gobernabilidad de la educación nació asociada a la necesidad de reforzar su comportamiento sistémico. De este modo, para Tenti el gobierno supone la noción de sistema, es decir, algo que tiene una consistencia, una realidad, y que se deja gobernar; pero también la idea de que existen actores dotados de intereses, proyectos, programas, recursos y fuerza para darle una dirección y sentido a dicho sistema (TENTI, 2004). Otra afirmación que da cuenta de la pertinencia de la mirada sistémica para efectos de la gobernabilidad de la educación, es la que formula Delors (1996), quien refiere que en las sociedades futuras, la necesidad de movilizar fuerzas mucho más allá de las instituciones formales conferirá a los poderes públicos un nuevo papel. Por una parte, tendrán que garantizar la visibilidad y la legibilidad del sistema educativo, asegurando así la estabilidad del conjunto. Por otra, deberá suscitar asociaciones para liberar nuevas energías para la educación.

En este orden de ideas, el problema de la conducción de los sistemas educativos, tiene que ver con el hecho de que la educación cada vez se parece menos a un sistema, no tiene un centro desde donde se lo conduzca, los protagonistas son cada vez más numerosos y el juego que mantienen -alianzas, obstáculos- es cada vez más complicado y en muchos casos más polarizado (TENTI, 2004). Por su parte, Tedesco agrega que la gobernabilidad de los sistemas educativos debe colocarse en el marco más general de los problemas de gobernabilidad de la sociedad. Existen numerosas evidencias que justifican esta mirada más amplia, numerosos fenómenos que ocurren fuera del sector educativo pero que penetran en su esfera de acción (TEDESCO, 2004).

Entre las situaciones que han venido a complicar el gobierno de la educación queremos poner énfasis en el hecho de que actualmente los sistemas educativos se 
encuentran fragmentados, son cada vez más policéntricos y que los actores que juegan en el campo de la política educativa son más numerosos porque a la comunidad escolar tradicional, se suman las asociaciones profesionales, empresarios, sindicatos, medios de comunicación, organismos internacionales, expertos y por supuesto las instancias locales de gobierno.

En el caso de México, partimos de la hipótesis de que el Federalismo educativo que se instaura a través del Acuerdo Nacional para la Modernización de la Educación Básica, ha afectado la gobernabilidad del sistema educativo, particularmente la capacidad de conducción de la Secretaría de Educación Pública como autoridad nacional encargada de fijar los objetivos, estrategias y líneas de acción de la política educativa general. Resulta indispensable contextualizar esta tarea de conducción, señalando que tiene que ver un sistema con más de 37 millones alumnos, 240 mil escuelas y un millón 600 mil maestros (INEE, 2008). Sistema que al estar organizado federalmente, se compone por 32 subsistemas educativos locales con centros de autoridad propios.

\section{Política educativa nacional y federalismo educativo}

Una vez planteada la cuestión del gobierno del sistema educativo como un problema político, teórico, y práctico, nos ocuparemos ahora del significado que la noción de política educativa nacional tiene en el contexto del actual modelo de organización y funcionamiento de la educación en México.

La comprensión de este modelo y del papel que ha venido desempeñando el gobierno federal a través de la Secretaría de Educación Pública, exige que hagamos antes un repaso general a las etapas de gestión que le antecedieron: desconcentración y descentralización. A fin de ilustrar esquemáticamente los procesos de reforma de que ha sido objeto la organización del sistema educativo mexicano, y con ello poner en perspectiva el Federalismo educativo, como esquema de operación vigente, nos apoyaremos en el cuadro 1. 
Cuadro 1 - Variantes del proceso de reforma del sistema educativo en México

\begin{tabular}{|c|c|c|c|c|}
\hline $1921 \ldots$ & $1978 \ldots$ & $1983 \ldots$ & \multicolumn{2}{c|}{$1992 \ldots$} \\
\hline \multicolumn{2}{|c|}{$\begin{array}{c}\text { GOBIERNO FEDERAL } \\
\text { Secretaría de Educación Pública (SEP) }\end{array}$} & GOBIERNO FEDERAL (SEP) & $\begin{array}{c}\text { GOBIERNO } \\
\text { FEDERAL (SEP) }\end{array}$ & $\begin{array}{c}\text { GOBIERNOS } \\
\text { ESTATALES }\end{array}$ \\
\cline { 2 - 4 } Centralización (I) & Desconcentración (II) & "Descentralización” (III) & \multicolumn{2}{|c|}{ "Federalismo educativo" (IV) } \\
\hline $\begin{array}{c}\text { Representaciones } \\
\text { de las oficinas de la } \\
\text { SEP en los Estados }\end{array}$ & $\begin{array}{c}\text { Delegaciones de la } \\
\text { SEP en los Estados }\end{array}$ & $\begin{array}{c}\text { Servicios Coordinados de } \\
\text { Educación Pública en los Estados }\end{array}$ & $\begin{array}{c}\text { Facultades } \\
\text { normativas a } \\
\text { nivel nacional }\end{array}$ & $\begin{array}{c}\text { Facultades } \\
\text { operativas en el } \\
\text { Estado }\end{array}$ \\
\hline
\end{tabular}

Fuente: Covarrubias (2000).

Como se observa en el cuadro, en la gestión del sistema educativo en México pueden distinguirse cuatro grandes etapas. La primera (I), corresponde a la fase de Centralización que inicia con la creación de la Secretaría de Educación Pública en 1921 y con ello, el despliegue de la acción educativa del Estado a gran escala. La actuación de los sucesivos gobiernos habría de caracterizarse por mantener y extender los servicios educativos. Junto a la expansión cuantitativa del servicio educativo que pretendió llegar a todas las regiones del país, se consolidó paralelamente una estructura en que las decisiones sustantivas se definieron y ejercieron desde el centro del país.

La injerencia federal se consideró como condición indispensable del crecimiento educativo, solo el gobierno nacional podía garantizar los recursos suficientes para ampliar la matrícula escolar, meta vinculada al desarrollo económico y social del país. Refiriéndose a la naturaleza centralista de este proceso, Aguilar apunta que en el siglo XX, la configuración postrevolucionaria del sistema político y la necesidad de modernizar la vida social y económica del país acentuaron el predominio del gobierno federal sobre los otros órdenes de gobierno (AGUILAR, 1996). Luego de sostener que la centralización es una tendencia natural de los sistemas federales, agrega que en el caso del federalismo mexicano, dio como resultado la supremacía del gobierno federal sobre las soberanías de las entidades federativas. De este modo, sostiene que el predominio del poder federal fue la única posibilidad de hacer compatible el desarrollo económico con las exigencias de justicia social, trayendo consigo la expansión del aparato estatal al fortalecerse su intervención en las áreas de beneficio social. Sin embargo, la centralización también trajo consigo procesos de burocratización, problemas de eficiencia y eficacia del aparato administrativo, así como de calidad, eficiencia e inequidad de los propios servicios educativos.

La segunda etapa (II) conocida como Desconcentración comprende de 1978 a 1982, y tiene como antecedentes el crecimiento explosivo de la población y la masificación 
de la enseñanza, que derivaron en una expansión desordenada del sistema educativo, en la pérdida gradual y progresiva del control administrativo, así como en el deterioro de los aspectos sustanciales. El sistema educativo terminó por favorecer a las regiones más pobladas en perjuicio del medio rural. Urgía frenar el creciente distanciamiento entre las distintas zonas del país y propiciar una distribución más equitativa de las oportunidades educativas. A través de la desconcentración, el Gobierno Federal estableció en 1978 en cada uno de los estados de la República, órganos denominados Delegaciones Generales dependientes de la Secretaría de Educación Pública, agrupando en ellas todas las tareas operativas con las facultades de decisión respectivas con el propósito de que éstas se tomaran de acuerdo a las características particulares de las entidades federativas. Con la desconcentración se redistribuyó el poder de decisión entre los niveles e instancias de la propia Secretaría y representó una fase previa del proceso de descentralización educativa que habría de intentar el gobierno a partir de 1982.

La tercera etapa (III) en la gestión del sistema educativo en México, abarcó de 1982 a mayo de 1992 y fue conocida como “Descentralización”. Ésta se justificó en la búsqueda de una redistribución del poder de decisión más equilibrada para la prestación de los servicios en las entidades federativas; por el acercamiento de las decisiones al lugar donde se prestan los servicios; por la posibilidad de una mayor participación de la comunidad usuaria del servicio en la definición de necesidades a ser atendidas por los programas de trabajo; así como por la relevancia del aprendizaje a través de la participación de los actores en la definición de los contenidos curriculares. En su momento, la intención de descentralizar, se reflejó en el Plan Nacional de Desarrollo 1983-1988, donde se afirmaba que, la descentralización de la educación básica constituía una línea de acción fundamental para el mejoramiento de la eficiencia y calidad educativas y para auspiciar la participación de la comunidad (MÉXICO, 1983b). No obstante que a este periodo se le conoce como "descentralización educativa", en la práctica estuvo muy lejos de significar una verdadera redistribución del poder de decisión entre federación y estados. En su lugar, se optó por establecer en cada estado órganos denominados Servicios Coordinados de Educación Pública, mediante los cuales se buscó administrar los servicios educativos a través de un esquema de corresponsabilidad entre autoridades federales y locales. Sin embargo, el gobierno federal no sólo no transfirió los servicios, sino que además sus facultades normativas sobre un importante repertorio de aspectos de orden pedagógico y administrativo resultaron fortalecidas.

Finalmente, la cuarta etapa (IV) hasta ahora vigente, inicia formalmente en mayo 
de 1992 con la firma del Acuerdo Nacional para la Modernización de la Educación Básica y corresponde al llamado Federalismo educativo. Para Moctezuma (1993), la federalización es cualitativamente diferente a la descentralización que en otros países, ha significado la fragmentación de un sistema nacional o la simple transferencia de funciones y recursos. Afirma que en el caso de México, la federalización educativa fortalece tanto las facultades de la autoridad federal para garantizar una educación de unidad nacional, así como la participación de los gobiernos de los estados en la operación de los servicios. Según el discurso oficial, con el federalismo se replantearía la el papel de las administraciones educativas a nivel central y local; en el primer caso, para concentrar esfuerzos en el fortalecimiento de la capacidad de conducción nacional, en el segundo, para una mayor articulación entre el trabajo de la escuela, con autoridades, instituciones, y otros sectores de la comunidad (MÉXICO, 1992).

En nuestra opinión, el Federalismo educativo, alude a dos cuestiones: una, al hecho que la Federación transfiere a los gobiernos estatales, la administración de los servicios de educación básica y normal. Otra, que al circunscribirse la responsabilidad operativa del gobierno federal a la capital del país, la regulación general del sistema educativo se reafirma como competencia sustantiva de la Secretaría de Educación Pública. Con ello, se establece una delimitación más clara de atribuciones entre los órdenes de gobierno federal y estatal, situación que no ocurrió en la etapa previa, en donde ambas aparecían al menos en teoría, como responsables de la operación del servicio en una de las entidades federativas.

Hoy día, a 18 años de haberse implantado el federalismo educativo, los datos existentes indican que esta medida no se ha traducido en una mejor enseñanza ya que persisten importantes problemas de eficiencia terminal y de calidad. Al referirse a este hecho, Santizo (2009) que sostiene que una de las principales causas que han impedido el éxito de la descentralización en México, es que se delegaron responsabilidades pero no se dotaron los medios que permitieran a las autoridades locales y comunidades escolares aprovechar los espacios de decisión que se les otorgaron.

Es importante mencionar también, que la descentralización educativa en nuestro país, se ha enmarcado por un contexto de crisis económica recurrente que ha dado lugar a un deterioro de las condiciones de vida de un número cada vez mayor número de habitantes. Lo cual ha impedido que, a pesar de los esfuerzos que realizan las autoridades educativas, se puedan erradicar los problemas de analfabetismo, abandono, ausentismo, reprobación y bajo aprovechamiento. Por otro lado, perviven fenómenos como el 
centralismo, el burocratismo, la falta de actualización docente, una fuerte presencia de intereses sindicales, así como la deficiente formulación e implementación de políticas que afectan la gestión y funcionamiento del sistema educativo limitando las posibilidades de resolver los problemas que afectan a la educación.

No obstante, la descentralización también ha ofrecido oportunidades para que la educación se acerque más a las necesidades de las comunidades y escuelas. Diversas entidades federativas han realizado ejercicios a nivel regional, relativos al diseño de políticas educativas, de planes y programas de estudio, a la promoción de un nuevo liderazgo por parte de directivos escolares, al impulso al trabajo colegiado, así como a la difusión de proyectos como estrategias para resolver problemas pedagógicos en zonas escolares y escuelas (CALVO, 2002).

Es importante aclarar que cuando hablamos del federalismo educativo, no buscamos manifestarnos en contra del federalismo como mecanismo de gobierno, sino que nos referimos a la estrategia concreta de descentralización en México, que por la forma en que se realizó ha presentado desventajas y oportunidades.

Después de este examen sucinto de los periodos de gestión del sistema educativo, estamos en condiciones de precisar la noción de política educativa nacional en el contexto del Federalismo educativo hoy vigente. Para los propósitos de este ensayo, la definimos del modo siguiente: conjunto de objetivos, estrategias y líneas de acción que tienen que ver con la conducción y el desarrollo del sistema educativo en su conjunto.

Una política cuyo carácter nacional, procede no sólo del hecho de que sea promovida o realizada por la autoridad federal, sino porque está referida a un asunto que por principio, involucra por igual a autoridades estatales y locales. Política, que si bien como acción colectiva supone la intervención de un extenso número de participantes, se encuentra directamente asociada al quehacer sustantivo de la Secretaría de Educación Pública, en el sentido, que su papel como autoridad federal le impone en esta materia, responsabilidades en dos vertientes: una, relacionada con la conducción global del sistema educativo; otra, con el establecimiento de un marco de referencia para la planeación que corresponde realizar a las autoridades locales.

Este carácter rector del gobierno federal, tiene un amplio sustento en la legislación a través de diferentes ordenamientos. El artículo $3^{\circ}$ de la Constitución Política de los Estados Unidos Mexicanos, además de consagrar el derecho a la educación establece la obligatoriedad, gratuidad y el carácter laico de la educación que es impartida por el Estado (MÉXICO, 2002). 
Por su parte, la Ley General de Educación (MÉXICO, 1993) -reglamentaria de este precepto constitucional- al realizar la distribución de la tarea educativa, en el artículo 12 establece las atribuciones que corresponden de manera exclusiva al poder ejecutivo federal por conducto de la Secretaría de Educación Pública, entre éstas destacan:

- Determinar para toda la República los planes y programas de estudio para la educación preescolar, primaria, secundaria, normal y para la formación de maestros de educación básica;

- Realizar la planeación y programación globales del sistema educativo nacional; y

- Efectuar la evaluación del sistema educativo nacional así como, fijar los lineamientos generales de la evaluación que las autoridades locales deban realizar.

En su artículo 13, esta ley, señala también las facultades que corresponden a las autoridades educativas locales, resaltando la de prestar los servicios de educación inicial, básica, indígena, especial, así como la formación de profesores.

Considerando la naturaleza y alcance de las responsabilidades, en el sistema educativo mexicano pueden distinguirse cuatro niveles de administración: 1) Federal o central, 2) Estatal o subnacional, 3) Municipal o local y, el 4) Escolar.

Sin embargo, debe advertirse que la participación municipal se reduce a colaborar con el gobierno estatal respectivo, en la construcción, conservación y mejoramiento de edificios y equipos escolares. A manera de resumen, las responsabilidades de cada nivel se presentan en el cuadro 2. 


\section{Cuadro 2 - Los niveles de administración del sistema educativo en México}

\begin{tabular}{|c|c|c|}
\hline Niveles & Responsabilidades & $\begin{array}{c}\text { Estructuras } \\
\text { Administrativas }\end{array}$ \\
\hline \multirow{4}{*}{ Administración Central } & \multicolumn{2}{|l|}{ Ámbito: Sistema educativo nacional } \\
\hline & $\begin{array}{l}\text { Normatividad general del sistema educativo, } \\
\text { determinación de planes y programas de estudio } \\
\text { para la educación básica y normal, fijar requisitos } \\
\text { pedagógicos para la educación inicial y } \\
\text { preescolar, establecer el calendario escolar, } \\
\text { elaborar libros de texto gratuitos, autorizar los } \\
\text { libros de texto para primaria y secundaria, regular } \\
\text { el sistema nacional de créditos, de revalidación y } \\
\text { equivalencias, realizar la planeación, la } \\
\text { programación y la evaluación globales del sistema } \\
\text { educativo, y ejercer las atribuciones necesarias } \\
\text { con el objeto de garantizar el carácter nacional de } \\
\text { la educación básica y normal. }\end{array}$ & $\begin{array}{l}\text { Oficinas Centrales de la Secretaría } \\
\text { de Educación Pública (SEP) } \\
\text { (*) De acuerdo al transitorio cuarto } \\
\text { de la Ley General de Educación, } \\
\text { en tanto se concluye la } \\
\text { transferencia de estos servicios al } \\
\text { Gobierno del D. F., esta } \\
\text { responsabilidad en la Capital del } \\
\text { país, corresponderá a la SEP }\end{array}$ \\
\hline & \multicolumn{2}{|l|}{ Ámbito: Capital del País } \\
\hline & $\begin{array}{l}\text { Prestación de los servicios de educación inicial, } \\
\text { básica y especial. }(*)\end{array}$ & $\begin{array}{l}\text { Administración Federal de } \\
\text { Servicios educativos en el Distrito } \\
\text { Federal (Órgano desconcentrado } \\
\text { de la SEP) }\end{array}$ \\
\hline \multicolumn{3}{|c|}{ Ámbito: Entidades Federativas } \\
\hline $\begin{array}{l}\text { Administración } \\
\text { Estatal }\end{array}$ & $\begin{array}{l}\text { Titularidad administrativa en su territorio, prestar } \\
\text { los servicios de educación inicial, básica y normal, } \\
\text { proponer contenidos regionales a incluir en los } \\
\text { planes y programas de estudio, ajustar el } \\
\text { calendario escolar para cada ciclo lectivo, } \\
\text { revalidar y otorgar equivalencias de estudios de la } \\
\text { educación básica y normal, otorgar, negar y } \\
\text { revocar autorización a particulares para impartir } \\
\text { educación básica y normal. }\end{array}$ & $\begin{array}{l}\text { Secretarías de Educación Pública } \\
\text { Estatales ó unidades } \\
\text { administrativas equivalentes. }\end{array}$ \\
\hline
\end{tabular}

\section{Ámbito: Local}

Administración

Promover y prestar los servicios educativos de Ayuntamientos.

Municipal cualquier tipo o modalidad, participar en el mantenimiento y provisión de equipo básico a escuelas públicas.

\section{Ámbito: Centros Educativos}

Administración Escolar

Prestación directa de los servicios educativos

Organización escolar. Conformada por directivos, órganos colegiados, profesores, personal de servicios educativos complementarios y, de apoyo y asistencia a la educación.

Fuente: Elaboración propia con base en la Ley General de Educación (MÉXICO, 1993).

Del repertorio y naturaleza de facultades citadas, destacan las relacionadas con la planeación del sistema educativo. El sustento jurídico de la misma se conforma, además de artículo 12 de la Ley General de Educación -antes señalado-, por el artículo 21 de la Ley de Planeación que establece que las dependencias del Ejecutivo Federal deberán planear y conducir sus actividades con sujeción a los objetivos y prioridades de la planeación 
nacional del desarrollo (MÉXICO, 1983a). Sin embargo, conviene hacer notar que aunque la Ley General atribuye la planeación general del sistema educativo al gobierno federal, la descentralización obliga a repensar esta función tanto en sus métodos como en sus recursos. Al incrementarse la intervención de las autoridades locales en la prestación de los servicios a partir del Federalismo educativo, la política educativa no puede seguir siendo considerada como una responsabilidad exclusiva del gobierno federal.

Una vez definidos la noción de política educativa nacional y el papel que como autoridad federal atañe a la Secretaría de Educación Pública, toca ahora indicar que dicha política se encuentra formalmente expresada en el programa sectorial que, de acuerdo a la Ley de Planeación antes mencionada, debe integrarse de manera cada seis años, que es la duración del periodo de gobierno federal en México. Así, por ejemplo, durante el periodo que corresponde al federalismo educativo, se han elaborado los siguientes:

- Programa de Desarrollo Educativo 1995-2000

- Programa Nacional de Educación 2001-2006

- Programa Sectorial de Educación 2007-2012

El último de los programas, corresponde al gobierno en curso, es decir, a la Presidencia de Felipe Calderón Hinojosa. En dicho documento, se expresan los objetivos, las estrategias y las líneas de acción de las dependencias y organismos federales que pertenecen a este sector.

Al presentarlo, el Presidente Calderón (MÉXICO, 2007) señaló: “El Programa Sectorial de Educación, como el resto de los programas sectoriales, ha sido elaborado tomando como punto de partida la Visión México 2030 y el Plan Nacional de Desarrollo, así como los resultados de una amplia consulta con actores relevantes del sector que han aportado elementos de diagnóstico y de acción.” Apuntó también, que su elaboración se apegó a los lineamientos de la Ley de Planeación, y que forma parte de una estrategia tanto para impulsar el desarrollo del país.

Concluimos este apartado, puntualizando que el Programa Sectorial de Educación representa para nuestros propósitos, la expresión actual del proceso de cambio o devenir de la política educativa al que aludimos con el título de nuestro artículo, y que se pone de manifiesto con el Federalismo educativo, ya que de un sistema relativamente uniforme regulado por una autoridad central, se transita a otro pluricéntrico, fragmentado en 32 subsistemas educativos locales. Ocasionando que la política educativa deje de ser asunto exclusivo del gobierno federal. 
Esto ocurre al lado de una mayor democratización y pluralidad política, que hace que nuevos gobiernos de diferentes niveles emerjan de partidos de oposición. Lo cual, sin duda, torna más difícil la tarea gubernamental dado que se deben consensuar decisiones, tomar en cuenta puntos de vista de diferentes partidos políticos, grupos sociales y grupos disidentes con proyectos muchas veces contrapuestos. Conviene señalar que en 1989, un partido de oposición logra la primera gubernatura y que diez años después ya sumaban 11, incluyendo al Distrito Federal como la capital del país. De estas, seis corresponden al Partido Acción Nacional, cuatro al Partido de la Revolución Democrática y una para una alianza entre diversos partidos (ESPINOZA, 2002). Con ello, la progresiva presencia de poderes políticos relativamente autónomos, que no son responsables ante el gobierno central, sino ante sus electores introduce una nueva dinámica en el devenir de las políticas públicas en general, y de la educativa en lo particular. Con el federalismo, la formulación e implementación de la política educativa se han vuelto cada vez más complicadas. La presencia de nuevos actores y centros de decisión a nivel local, obliga a mayores y más intensas negociaciones para articular y obtener el apoyo requerido para una línea de actuación o de reforma. En este contexto, emerge el problema de la capacidad de gobierno del sistema educativo mexicano y el significado que el Programa Sectorial de Educación 2007-2012 formulado por la Secretaría de Educación Pública como ministerio nacional, tiene como expresión de la política educativa nacional.

\section{Los problemas de articulación de la política educativa en el federalismo}

Como hemos podido ver, desde la firma el Acuerdo Nacional para la Modernización de la Educación Básica en mayo de 1992, la política educativa cambia de genealogía. Los objetivos, estrategias y metas antes considerados como nacionales, empiezan a alinearse a las necesidades -y sobre todo a ajustarse a las capacidades- de cada estado. A partir de entonces afloran y adquieren preponderancia los problemas de sintonía; la política educativa como había sido concebida hasta ese momento se quiebra. Las prioridades y metas enunciadas en dicho documento, pasan en estricto sentido, a tener carácter obligatorio sólo para las unidades responsables del ministerio de educación, y los organismos agrupados en el sector de la administración pública federal coordinado por dicha dependencia.

Proceso sin duda significativo, porque da lugar a una suerte de escisión de la planeación educativa que pierde cohesión. Lo que obedece no tanto a que las autoridades educativas locales asumieran o ejercieran diligentemente las competencias transferidas, 
sino como a factores asociados al hecho de que los gobiernos subnacionales y locales poseen sus propias tradiciones, "miradas", formas de entender o hacer las cosas; así como a que éstos, cuentan con diferentes capacidades administrativas para operacionalizar políticas y programas, razón por la cual, se registran trayectorias y logros disímiles en cada caso (COVARRUBIAS, 2006).

Sin embargo, de los distintos factores que afectan la gobernabilidad del sistema educativo en este ensayo nos centramos en el problema de la vertebración de la política en el federalismo. Al respecto, aportamos dos tipos de evidencias que nos parecen significativas. Una, la serie de testimonios de los secretarios de educación de los distintos estados de la República Mexicana, y funcionarios de la Secretaria de Educación Pública, vertidas en una serie de reuniones denominadas: Diálogos Nacionales de Política Educativa. Y dos, una cuestión de fondo: la forma en que se encuentran estructurados los periodos de gobierno a nivel federal y local en el Estado mexicano.

En el primer caso, son ilustrativas no solo las propias reuniones en sí, sino sobre todo lo que en ellas expresaron los responsables de la educación en los estados con respecto a los problemas de articulación entre los programas federales y locales en la materia. Para empezar sería conveniente decir que la información que aquí se presenta no tuvo una difusión precisamente pública, se trata de las actas o lo que se conoce como "resúmenes ejecutivos". En dichos documentos, se define a estas reuniones como pequeños grupos de trabajo, que bajo una "mecánica estrictamente confidencial”, permiten la discusión de los nuevos escenarios para la gestión del sistema educativo mexicano con los pares estatales y los subsecretarios de; Planeación y Coordinación y de Educación Básica y Normal de la Secretaría de Educación Pública (MÉXICO, 2003). Entre sus objetivos se mencionan:

- Crear grupos operativos de trabajo entre los Secretarios de Educación de los distintos estados y la Secretaría de Educación Pública.

- Confrontar experiencias y políticas puestas en marcha por los distintos estados, y a partir de las mismas, evaluar alternativas.

- Evaluar estrategias cooperativas para el mejoramiento de los sistemas educativos de cada uno de los estados.

La estrategia seguida para que los secretarios de educación de las entidades federativas se integraran en grupos pequeños los cuales permitieran el debate amplio sobre los siguientes temas: Calidad, Equidad, Federalismo y Financiamiento. Para ello se organizaron cuatro grupos regionales de trabajo: 


\begin{tabular}{|c|c|}
\hline Región Noroeste & Región Noreste \\
\hline $\begin{array}{ll}- & \text { Baja California. } \\
\text { - } & \text { Baja California Sur. } \\
- & \text { Chihuahua. } \\
- & \text { Colima. } \\
- & \text { Jalisco. } \\
- & \text { Sonora. } \\
- & \text { Nayarit. } \\
- & \text { Sinaloa. } \\
\end{array}$ & $\begin{array}{ll}\text { - } & \text { Aguascalientes. } \\
- & \text { Coahuila. } \\
\text { - } & \text { Durango. } \\
\text { - } & \text { Hidalgo. } \\
- & \text { Nuevo León. } \\
- & \text { San Luis Potosí. } \\
- & \text { Tamaulipas. } \\
- & \text { Zacatecas. }\end{array}$ \\
\hline Región Centro & Región Sureste \\
\hline $\begin{array}{ll}\text { - } & \text { Distrito Federal. } \\
\text { - } & \text { Morelos. } \\
\text { - } & \text { Michoacán. } \\
\text { - } & \text { Guanajuato. } \\
\text { - } & \text { Guerrero. } \\
\text { - } & \text { Estado de México. } \\
\text { - } & \text { Tlaxcala. } \\
\text { - } & \text { Puebla. } \\
- & \text { Querétaro. }\end{array}$ & $\begin{array}{ll}\text { - } & \text { Campeche. } \\
\text { - } & \text { Oaxaca. } \\
\text { - } & \text { Quintana Roo. } \\
\text { - } & \text { Tabasco. } \\
\text { - } & \text { Veracruz. } \\
\text { - } & \text { Yucatán. } \\
\text { - } & \text { Chiapas. }\end{array}$ \\
\hline
\end{tabular}

1. Los temas abordados se agruparon en cuatro vertientes: Calidad, Equidad, Federalismo y Financiamiento. El procedimiento que utilizamos consistió en analizar los informes que dan cuenta de los temas abordados y acuerdos de cada grupo de trabajo, con el propósito de identificar específicamente las declaraciones relacionadas con la necesidad de mejorar la coordinación entre la planeación de la autoridad federal y de las autoridades estatales. En este sentido, es importante señalar que el tema formó parte de la agenda de los cuatro foros regionales. Las opiniones o reclamos que al respecto expresaron los secretarios de educación locales a funcionarios de la Secretaría de Educación Pública del gobierno federal, se pueden sintetizar en los siguientes cinco puntos: Es necesario mejorar la articulación entre el programa nacional y los programas estatales de educación en sentido bidireccional, tanto en la fase de diseño como en la implementación.

2. Se requiere institucionalizar mecanismos de planeación y coordinación conjunta entre autoridades educativas federales y locales. Se menciona la necesidad de contar con un órgano colegiado en el que los actores discutan qué es lo que cada uno quiere hacer y de qué manera se pueden apoyar.

3. El programa nacional de educación se formula de manera unilateral y centralizada por lo que las metas federales no responden a las necesidades locales. Se pone en entredicho la contribución de los proyectos federales a la calidad de la educación a nivel local.

4. El programa nacional impone cargas de trabajo adicionales a las autoridades estatales sin proveerles del respaldo financiero adecuado. Existe la percepción en los funcionarios locales de que sus estados sólo aportan recursos a los programas federales, por lo que proponen revisar también las necesidades financieras de los programas de los estados.

5. Los estados disponen de un margen de maniobra estrecho provocado por la insuficiencia crónica de recursos financieros. Si bien reciben fondos del gobierno federal éstos se deben destinar al pago de nómina así como a la realización de los programas federales en la entidad federativa. 
En la información presentada saltan a la vista coincidencias en lo que expresan los responsables de la educación de los estados con relación a la necesidad de articular las políticas federal y estatales. No los citamos por razones de espacio, pero también abundan los reclamos sobre el financiamiento. Los cuales además de estar anudados al problema de la articulación de las políticas, nos hablan del limitado margen de maniobra de que disponen las autoridades educativas tanto a nivel federal como local para invertir en nuevos proyectos. Resulta expresiva la afirmación de responsables de la educación en los estados de la Región Noreste en el sentido de que aproximadamente el 95\% del presupuesto lo deben destinar al pago de personal.

También conviene apuntar, que en diciembre de 2005 se realizó la Primera Reunión Nacional de Autoridades en Materia de Planeación y Evaluación Educativa en la que se determinó proponer al Consejo Nacional de Autoridades Educativas la creación de una instancia permanente para atender exclusivamente los temas relacionados con la planeación educativa. Meses después, el 3 de abril de 2006, se establece formalmente la Comisión de Trabajo de Autoridades Educativas Responsables de la Planeación y Evaluación, como "una instancia deliberativa y temática", integrada por los titulares de las áreas de planeación y evaluación de las secretarías e institutos de educación de las entidades federativas, bajo la coordinación de la Unidad de Planeación y Evaluación de Políticas Educativas de la Secretaría de Educación Pública (CONAEDU, 2006). Esto con un propósito nada sencillo: propiciar la articulación de la planeación del Sistema Educativo Nacional.

Los temas que afectan a la gestión federalizada del sistema educativo, también han sido abordados en la Conferencia Nacional de Gobernadores -creada en 13 de julio de 2002-, que es un espacio de diálogo e interacción entre los Titulares de los Ejecutivos Estatales y el Gobierno Federal Mexicano. De una revisión de los acuerdos emanados de las diferentes reuniones de gobernadores sobre la materia que nos ocupa, consideramos necesario citar los siguientes por estar relacionados específicamente con la coordinación de la política educativa: 


\section{REUNION}

XVII Reunión Ordinaria celebrada el 7 de junio de 2004 en Villahermosa, Tabasco (CONAGO, 2004).

XXIX Reunión Ordinaria celebrada el 11 de septiembre de 2006 en Nuevo Vallarta, Nayarit (CONAGO, 2006).

XXXI Reunión Ordinaria celebrada el 16 de febrero de 2007 en Tlaxcala, Tlaxcala (CONAGO, 2007).

ACUERDOS

Se aprueba la propuesta de realizar una reunión con el Secretario de Educación, y que los Secretarios de Educación de las Entidades Federativas mantengan contacto permanente debido a que la información que presenta la Comisión difiere de lo que sucede en algunos Estados en esta materia.

La Gobernadora del Estado de Zacatecas, Amalia D. García Medina, plantea en el seno de la reunión que la CONAGO se manifieste porque la Federación agilice la entrega de recursos a los Estados para educación.

En este evento, la Secretaria de Educación Pública del Gobierno Federal, Josefina Vázquez Mota, propuso establecer una agenda educativa conjunta entre la Conferencia Nacional de Gobernadores y la Secretaría de Educación Pública, misma que contendría los siguientes doce puntos:

Rezago educativo

Capacitación y actualización de los maestros

Participación de la comunidad escolar

Evaluación para la calidad educativa

Conformación de un sistema nacional de información educativa

Modernización tecnológica para la educación

Integralidad en la educación media superior

Seguridad y certeza

Infraestructura

Certificación de instituciones educativas

Descentralización educativa

Gobernabilidad.

XXXII Reunión Ordinaria celebrada el 29 de mayo de 2007 en Puerto Vallarta, Jalisco (CONAGO, 2007)

Se autoriza al Coordinador de la Comisión de Educación de la CONAGO, a reunirse con el Secretario de Hacienda y Crédito Público para revisar los aspectos presupuestales, destacando la actual insuficiencia de recursos para infraestructura y para cubrir necesidades indispensables.

Fuente: Elaboración propia con base en las declaratorias de las 36 Reuniones de la CONAGO (2002, 2004a , 2004b, 2006, 2007a, 2007b).

Conviene señalar que como resultado de la propuesta hecha por la Secretaría de Educación Pública, Josefina Vázquez Mota en la XXI Reunión Ordinaria de la CONAGO, que se enuncia en el cuadro anterior, los días 26 y 27 de abril del 2007 se efectuó en Saltillo, Coahuila, la Reunión Nacional de Secretarios de Educación. ${ }^{3}$ Según el informe oficial (CONAGO, 2007), las discusiones que tuvieron lugar en la línea temática del Federalismo educativo, se centraron en la "Gobernabilidad y financiamiento y en la Coordinación entre los tres niveles de gobierno". En la reunión, se abordó también la revisión del Acuerdo Nacional para la Modernización de la Educación Básica y Normal de 1992 y se propuso la elaboración de un nuevo Acuerdo Nacional. Entre los aspectos más trascendentes, que se señalan para el diálogo sobre un Nuevo Acuerdo, se mencionaron los siguientes:

3 La reunión se llevó a cabo en dos partes. La primera, consistió en la realización en seis mesas de trabajo, donde se presentaron los diagnósticos estatales, encuadrados dentro de ocho líneas temáticas: 1) Financiamiento educativo, 2) Abatimiento a los rezagos, 3) Ampliación de servicios educativos, 4) Calidad educativa, 5) Participación social en la educación, 6) Federalismo educativo, 7) Salarios y prestaciones y 8) Evaluación del sistema educativo. En la segunda, realizó una revisión del Acuerdo Nacional para la Modernización de la Educación Básica y Normal de 1992. 
- Ampliación del Acuerdo a todos los niveles educativos, impulsar una reforma estructural en la materia que integre a todos los niveles educativos;

- Redefinir las responsabilidades en materia de distribución, asignación y uso de recursos financieros de los tres órdenes de gobierno, previendo alternativas para compensar el déficit financiero existente;

- Contenidos, materiales, y evaluaciones para mejorar la calidad educativa,

- Ampliación de los servicios e infraestructura educativa para garantizar la cobertura universal de la educación; y

- Promover la participación de padres de familia y otros actores de los ámbitos público, privado y social en el proceso educativo y su mejora.

Corresponde ahora referirnos al segundo tipo de evidencias, que en nuestra opinión, tiene que ver con una causa de fondo de la desarticulación e incoherencia de las acciones del gobierno mexicano en las grandes cuestiones sociales como la educación. Los múltiples "desfases" derivados de la asincronía entre los ciclos de actividad de las administraciones públicas es origen estructural de importantes incoherencias entre la planeación nacional y la local.

Como lo señalamos en el punto 1, donde planteamos el gobierno del sistema educativo como una tarea compleja, el sistema educativo no opera en el vacío, en el caso de México, los problemas de articulación a los que nos hemos referido hay que situarlos en el marco de lo que apunta Aguilar (2010), en el sentido de que hoy, una cuestión crítica de la operación del gobierno es su fragmentación e incoherencia. Sostiene que el Estado mexicano es una constelación de organismos autorreferidos y políticas divergentes. A la vista está la desarticulación de conceptos, enfoques, prácticas y prioridades. Por nuestra parte, añadiríamos que la política educativa es uno de los botones de muestra de ello.

Como se muestra en el siguiente cuadro 3, sólo cinco periodos de gobierno de las 32 entidades federativas, coinciden con el ciclo del gobierno central: Chiapas, Distrito Federal, Guanajuato, Morelos y Tabasco. Los restantes veintisiete estados, deben desarrollar sus programas, teniendo en cuenta dos diferentes programas nacionales de educación. Por ejemplo, en el caso de Aguascalientes, el "gobierno estatal entrante" debe elaborar su planeación teniendo en cuenta las pautas de un "gobierno federal saliente". En el cuadro también se muestra la filiación política de los gobiernos. Como puede verse, el partido en el gobierno federal es el Partido Acción Nacional (PAN), y los partidos de oposición son el Partido Revolucionario Institucional (PRI) y el Partido de la Revolución Democrática (PRD).

Lo anterior, explica en gran medida la inquietud expresada por el entonces Subsecretario de Educación Básica y Normal, Lorenzo Gomez-Morín en el sentido de que: 
“[...] hay estados que armaron su política con un plan nacional, y si hubo un cambio nacional, les cae otro programa (nacional) encima." (CONAEDU, 2003).

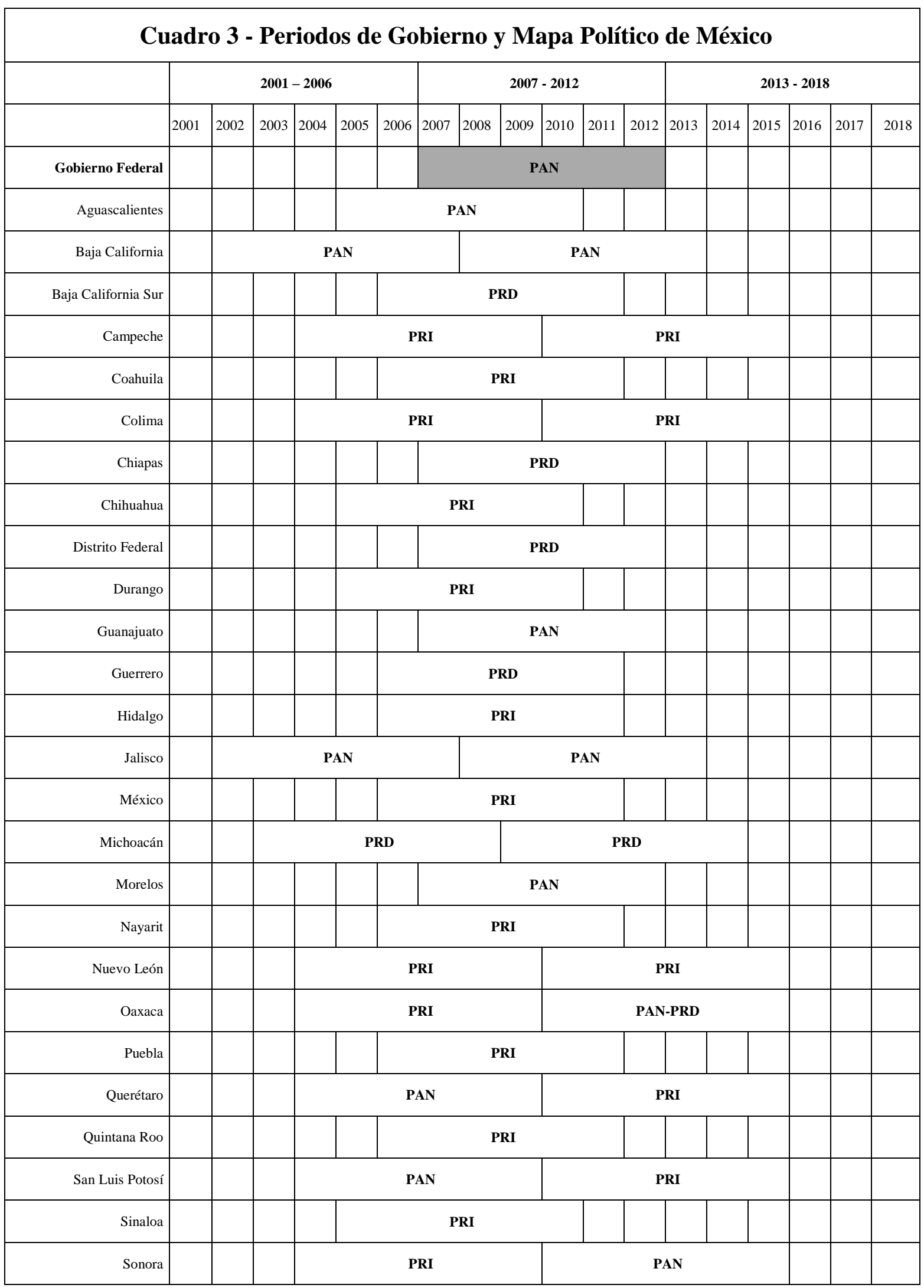




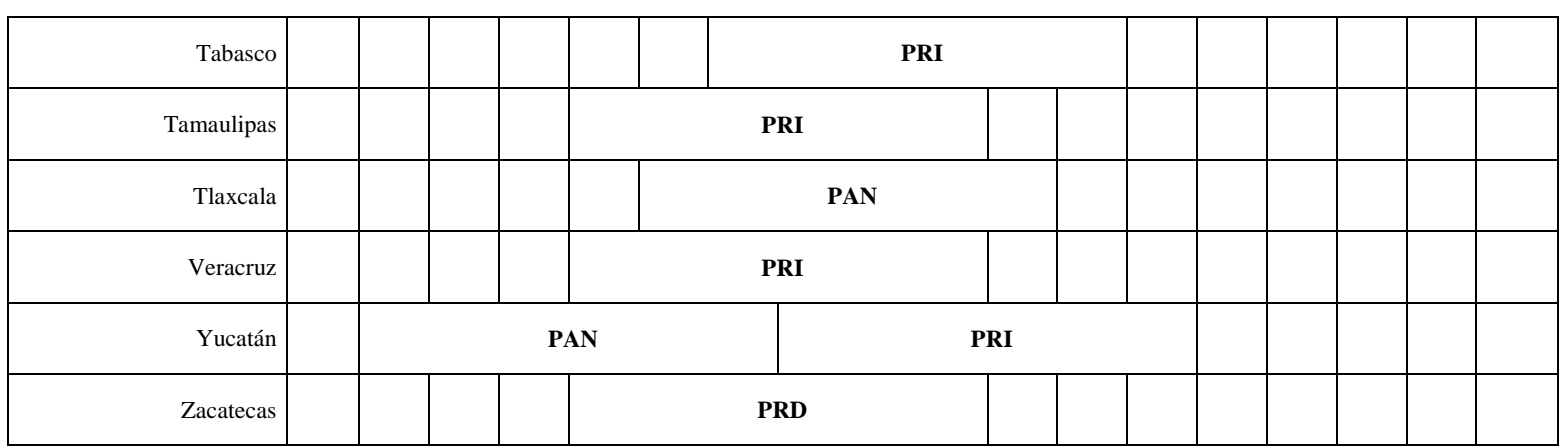

Siglas: Partido Revolucionario Institucional (PRI). Partido Acción Nacional (PAN). Partido de la Revolución Democrática (PRD).

Fuente: Elaboración Propia.

Si revisamos las cosas con mayor detalle, podemos percatarnos de que además de los lapsos o discontinuidades provocadas por los múltiples desfases, en el primer año de la administración federal se carece de una planeación nacional. Conviene apuntar que el Artículo 21 de la Ley de Planeación, otorga un plazo de seis meses para la elaboración del Plan Nacional de Desarrollo (MÉXICO, 1983b). Y que prácticamente los otros seis meses del primer año de gestión se destinan a la elaboración de los respectivos programas sectoriales. Esto es bastante esquemático, pero permite ilustrar el problema de la desincronización entre los periodos o ciclos de gobierno en México.

El cambio de gobierno federal da lugar a una cascada de transiciones a nivel local. Donde aparte existe el riesgo de transiciones de mayor duración, en razón de que las autoridades entrantes deben esperar además, a que el gobierno federal de a conocer el Plan Nacional de Desarrollo, y especialmente los distintos programas sectoriales que sirven de guía en la formulación de los propios planes y programas estatales. Esto habla de una dispersión de esfuerzos, se generan importantes costos de oportunidad.

Todo esto debe llevar a preguntarnos acerca de las implicaciones que la falta de sintonía en los periodos gubernamentales tiene, en términos de la planeación en general del desarrollo del país, así como de las propias entidades federativas, especialmente en rubros como el educativo que reclaman el tratamiento de políticas de largo plazo. Es muy complicado alinear las políticas cuando la mayoría de los estados deben armar su planeación teniendo como marco de referencia programas sectoriales de dos administraciones federales distintas. Al referirse a la necesidad de revisar cuidadosamente el sistema político mexicano para detectar disfuncionalidades, Francisco Casanova (2008) afirma que es conveniente reordenar los calendarios electorales y de los periodos de gobierno del país para mejorar la gobernabilidad y eficiencia. 
Aunado al desfase entre el diseño de la política nacional y las políticas estatales, la insuficiencia crónica de recursos agrava el hecho de que la SEP disponga de un margen de maniobra bastante estrecho para gravitar como elemento normativo del sistema de educación. Para ilustrar esta situación, es de hacer notar que México dedica el 5.7\% de sus ingresos nacionales a la educación con lo que porcentualmente tiene la mayor tasa de inversión en los países de la OCDE (21.7\% del gasto público total en comparación con un promedio de $13.3 \%$ en la OCDE), sin embargo, en números absolutos, el gasto por alumno permanece muy bajo. Por ejemplo, México destina 2,111 USD por alumno de educación primaria comparado con un promedio de 6,741 USD en la OCDE, y 2,236 USD por alumno de educación secundaria contra un promedio de 8,267 en la OCDE (2010). Además de lo limitado del presupuesto, más del $90 \%$ de los gastos es consumido por el pago de nómina personal (OECD, 2010).

De continuar la misma estructura de gasto, es decir, aumentos atados principalmente a gasto corriente, no está claro de dónde va a salir el financiamiento adicional para destinarlo por ejemplo a programas especiales de calidad, equidad y eficiencia de la educación. De los que en gran medida dependen las posibilidades de influir en el futuro en la dirección deseada.

En suma, el impacto de las múltiples transiciones de gobierno no es fácil de medir, estamos hablando de políticas de mediano y largo plazo, pero como ya lo hemos hecho notar, los desfases de la política educativa perjudican seriamente la dinámica y la eficiencia del gobierno en esta importante área.

\section{Programa sectorial de educación versus política nacional de educación}

En este apartado realizaremos algunas precisiones que nos sirvan para responder a la pregunta de si en las condiciones que mencionamos anteriormente, el Programa Sectorial de Educación y la Política Nacional de Educación son cuestiones equivalentes. Para empezar debemos decir que, si bien en un sentido amplio en el concepto de sector pueden quedar comprendidas todas las organizaciones públicas y privadas que dentro de una sociedad proporcionan un determinado bien o servicio; específicamente en el campo de la administración pública, un sector alude a una de las partes en que ésta se divide para cumplir con una función o propósito que es inherente al Estado. La sectorización representa la delimitación de funciones y competencias en grupos homogéneos de actividad para precisar responsabilidades, evitar duplicidad de funciones y permitir que las decisiones gubernamentales fluyan de manera eficiente. 
En tal sentido, la política de un sector puede ser definida como la conducta que asume el gobierno con la intención de articular efectivamente las acciones de las dependencias y entidades que concurren a la ejecución de una misma materia, bajo la coordinación de un ministerio legalmente encargado de fijar las directrices y orientaciones necesarias para dar coherencia a las políticas gubernamentales y evitar con ello, la contradicción de acciones dentro de un mismo sector de actividad. Así, la acción del conglomerado de organizaciones que conforman un sector, gravita singularmente sobre un personaje que posee formalmente la autoridad y responsabilidad de formular la política sectorial: el ministro o secretario del ramo o sector.

En un sistema federal, al adoptar las administraciones públicas esta modalidad de organización da lugar a la existencia de sectores a nivel federal, estatal y municipal. Por supuesto que en el caso de la administración pública federal, y a diferencia del nivel local, los sectores se caracterizan porque sus actividades generalmente tienen repercusiones para el país en su conjunto. En este sentido, la formulación de la política sectorial a cargo de un ministerio federal, constituye una responsabilidad vinculada a la noción de política nacional, cuya expresión formal se halla contenida ordinariamente en los planes o programas sectoriales que por su naturaleza y esfera de influencia, “norman” al conjunto de programas sectoriales e institucionales de carácter local.

Estas precisiones, resultan útiles para deslindar la noción de política educativa nacional con respecto al Programa Sectorial de Educación 2007 2012. Planteando de entrada, que no son cuestiones equivalentes; la primera tiene como referente al sistema educativo como un todo e incumbe a los tres órdenes de gobierno. El Programa Sectorial, por su parte, aunque también tiene en la mira al sistema educativo nacional, sus objetivos, líneas de acción y metas comprometen en sentido estricto, únicamente a las unidades administrativas de la Secretaría de Educación Pública y a los órganos sectorizados coordinados por esta dependencia. Lo mismo ocurre, en cada entidad federativa, donde las autoridades locales se rigen, en primera instancia, por sus propios programas sectoriales de educación.

De igual manera, la propia denominación del Programa Sectorial, parece dar cuenta de una especie de renuncia o reconocimiento por parte del gobierno federal, de las acortadas posibilidades de conducción del sistema educativo nacional. El carácter sectorial del programa, contrasta con la designación dada a los programas precedentes, citados anteriormente.

Lo que en apariencia pudiera ser solo de una cuestión de forma, adquiere 
significado si se toman en cuenta las escasas referencias en el mismo, a la gestión y administración descentralizada del sistema educativo. Por ejemplo, en el Programa Nacional de Educación 2001-2006, se dedica una de sus tres partes, a la gestión del sistema educativo.

\section{Reflexiones finales}

Después de dos décadas de reformas a los sistemas educativos en diversos países de Iberoamérica, surge con claridad la cuestión del gobierno del sistema educativo como un problema teórico, político y práctico. Y dado que éste sistema no opera en el vacío, es un tema que debe situarse en el contexto más amplio de los problemas de gobierno de la sociedad en su conjunto. Existen numerosas evidencias que justifican ampliar la mirada, ya que de manera creciente fenómenos que ocurren fuera del sistema educativo penetran en su esfera de acción

Como lo hemos podido ver el problema de la conducción de los sistemas educativos, tiene que ver con el hecho de que la educación cada vez se parece menos a un sistema, carece de un centro único desde donde se lo conduzca, los actores son cada vez más numerosos, y su interrelación cada vez más complicada y en muchos casos más polarizada.

En México con el Acuerdo Nacional para la Modernización de la Educación Básica de mayo de 1992, mediante el cual se transfieren los servicios de educación básica a las entidades federativas, la política educativa pierde cohesión. La política compacta que había sido estructurada durante varias décadas de manera vertical inició a partir de entonces un proceso de desarticulación.

Entre las principales causas podemos mencionar que tuvo lugar una suerte de “desresponzabilización” por parte del gobierno federal. La descentralización fue utilizada para emprender un reparto de responsabilidades en el que, como si se tratara de un movimiento hidráulico, un nivel de gobierno recibe lo que el otro pierde. Como consecuencia, se descuidaron mecanismos que hubieran permitido articular esfuerzos entre los órdenes de gobierno.

No menos importante es la manera en que se encuentran estructurados los periodos de gobierno en el Estado mexicano. Como se ha podido documentar, se trata de una cuestión de fondo que limita la vertebración de la política en el federalismo. El desfase entre los ciclos gubernamentales a nivel federal y local, es origen de los problemas de fragmentación e incoherencia en la educación y en otras importantes áreas de política 
pública.

Frente a ello, un objetivo realista es el de construir con políticas comprehensivas de largo plazo que permitan sincronizar y reconstruir la unidad de acción del Estado en la educación y con ello aminorar los numerosos lapsus que se aquejan la acción en este campo. Un Estado desordenado, disperso, es ineficaz aun cuando disponga de recursos y detente formalmente el poder. Nuevas tendencias de gobierno y administración pública parecen dirigirse a la reconstrucción de la unidad perdida de la estructura y acción estatal.

En tal sentido, la reivindicación de una visión sistémica y comprehensiva de la tarea educativa en nuestro país, será necesaria ante el eventual riesgo de una mayor fragmentación del sistema educativo. Este enfoque resulta ser significativo no sólo para efectos del estudio del objeto, sino en términos de su gestión. Hoy, en que por la federalización un mayor número de instancias y actores se hallan involucrados en la tarea educativa, más necesaria resulta ser una visión que reconozca la existencia de los diferentes niveles de acción y la importancia de su articulación.

La segmentación del sistema educativo como efecto colateral de la descentralización, puede conducir a perder la perspectiva global esencial para la toma de decisiones, tanto a nivel estatal, como nacional, si no se toma en cuenta que los problemas educativos y más aún sus soluciones, superan las jurisdicciones y capacidades particulares.

En síntesis, con el desarrollo del presente ensayo, se espera poder aportar elementos que ayuden a pensar más claramente acerca de los problemas de desarticulación que afectan a las políticas públicas en México. Es un tema que hasta el momento, no ha suscitado mucho interés entre especialistas ni académicos. Sin embargo, en la estructura del Estado federal, es un factor que impide dar respuesta de una manera ordenada, coordinada y eficaz a las a los problemas que a todos nos afectan. 
ABSTRACT: The purpose of this article is to analyze the educational policy of Mexico educational policy from the signing of the Agreement for the Modernization of Basic Education in 1992 This reflection starts from the assumption that the model of educational decentralization or federalism that is established through the National Agreement has affected the governance of the education system as a whole, and in particular, the ability to drive the Ministry of Education as a national authority.

KEYWORDS: Federalism. Education policy. Decentralization. Governance. Education system. Sectoral policy.

\section{REFERENCIAS}

AGUILAR, L. El Estado fragmentado. Diario Reforma, Ciudad de México, p.17, 24 febr. 2010.

El federalismo mexicano: funcionamiento y tareas pendientes. In: HERNÁNDEZ, A. (Coord.). ¿Hacía un nuevo federalismo? México: Fondo de Cultura Económica, 1996. p.109-151.

CALVO PONTÓN, B. Introducción. In: CALVO PONTÓN, B. et al. La supervisión escolar de la educación primaria en México: prácticas, desafíos y reformas. Paris: UNESCO, 2002. p.21-41.

CASANOVA, F. Un laberinto sin minotauro. In: TORRES, E.; PINEDA, J. de D. (Coord.). Transición y nueva institucionalidad en México. México: Facultad de Estudios Superiores de la UNAM, LAT-NET, IAPEM, 2008. p.27-61.

CONAEDU. Acuerdo del Consejo Nacional de Autoridades Educativas para la formación de la Comisión de Trabajo de Autoridades Educativas Responsables de la Planeación y Evaluación. Campeche: Secretaría de Educación Pública, 2006.

. Diálogos Nacionales de Política Educativa. Acta región Noroeste. Culiacán: Secretaría de Educación Pública, 2003.

CONAGO. Acuerdos de la trigésimo primera reunión ordinaria de la Conferencia Nacional de Gobernadores. Tlaxcala, 2007a.

. Acuerdos de la trigésimo segunda reunión ordinaria de la Conferencia Nacional de Gobernadores. Puerto Vallarta, 2007b.

Acuerdos de la vigésimo novena reunión ordinaria de la Conferencia Nacional de Gobernadores. Nuevo Vallarta, 2006.

. Acuerdos de la décimo quinta reunión ordinaria de la Conferencia Nacional de Gobernadores. Ciudad Victoria, 2004a. 
. Acuerdos de la décimo séptima reunión ordinaria de la Conferencia

Nacional de Gobernadores. Villahermosa, 2004b.

Acuerdos de la segunda reunión ordinaria de la Conferencia Nacional de Gobernadores. Pachuca, 2002.

COVARRUBIAS, M. La coordinación de la política educativa en el sistema federal: la evaluación de la educación básica en México. México: Universidad Nacional Autónoma de México, 2006.

Federalismo y reforma del sistema educativo nacional. México: Instituto Nacional de Administración Pública, 2000.

CROZIER, M. EI fenómeno burocrático. Buenos Aires:Amorrortu, 1969.

DELORS, J. La Educación encierra un tesoro. Madrid: UNESCO-Santillana, 1996.

DICCIONARIO de la lengua española. Madrid: Real Academia Española, 2010.

ESPINOZA VALLE, A. Alternancia política y gobiernos locales en México. Estudios Sociológicos, México, v.10, n.1, p.67-89, 2002.

INSTITUTO NACIONAL PARA LA EVALUÁCION DE LA EDUCACIÓN [INEE]. Panorama educativo de México: indicadores del sistema educativo nacional. México: Secretaría de Educación Pública, 2008.

LUHMANN, N.; EBERHARD, K. El sistema educativo (problemas de reflexión). Guadalajara: Universidad de Guadalajara, 1993.

MAYNTZ, R. El Estado y la sociedad civil en la gobernanza moderna. Revista del CLAD Reforma y Democracia, [S.1.], v.21, p.1-8, 2001.

MÉXICO. Secretaría de Educación Pública. Programa sectorial de educación 20072012. México, 2007.

. Secretaría de Educación Pública. Diálogos nacionales de política educativa: actas y resumen ejecutivo. México, 2003.

. Constitución política de los Estados Unidos Mexicanos. México: Diario Oficial de la Federación, 2002.

Ley general de educación. México: Diario Oficial de la Federación, 1993.

. Secretaría de Educación Pública. Acuerdo nacional para la modernización de la educación básica. México, 1992.

Ley de planeación. México: Diario Oficial de la Federación, 1983a.

Poder Ejecutivo Federal. Plan Nacional de Desarrollo 1983-1988, el 31 de mayo de 1983. Diario Oficial de la Federación, México, 1983b. 
MOCTEZUMA, E. La educación pública frente a las nuevas realidades. México: Fondo de Cultura Económica, 1993.

OFFE, C. Modernity and the State: East, West. Cambridge: Polity Press, 1996.

ORGANISATION FOR ECONOMIC CO-OPERATION AND DEVELOPMENT

[OECD]. Education at a Glance. Paris, 2010.

ORGANIZACIÓN DE ESTADOS IBEROAMERICANOS [OEI]. Declaración de Concepción de la Organización de Estados Iberoamericanos para la Educación, la Ciencia y la Cultura. Concepción, 1996.

SANDER, B. La organización educacional como sistema social. La Educación, Washington, n.68/69, p.7-23, 1974.

SANTIZO, C. Escuelas y capital social en México. Metapolítica, México, n.64, p.5660, 2009.

TEDESCO, J. C. Prologo en gobernabilidad de los sistemas educativos en América Latina. Buenos Aires: UNESCO, 2004. p.11-13.

TENTI, E. Nuevos problemas de gobierno de la educación en América Latina. In: TENTI, E. (Coord.). Gobernabilidad de los sistemas educativos en América Latina. Buenos Aires: UNESCO, 2004. p.45-64. 